\title{
The Onset of Chaos in Spinning Particle Models
}

\author{
H. T. Chd* and J.-K. Kadi \\ Department of Physics, Tamkang University, \\ Tamsui, Taipei, Taiwan, Republic of China
}

(Dated: November 20, 2018)

\begin{abstract}
The onset of chaos in one-dimensional spinning particle models derived from pseudoclassical mechanical hamiltonians with a bosonic Duffing potential is examined. Using the Melnikov method, we indicate the presence of homoclinic entanglements in models with general potentials for the spins, and thus show that chaotic motions occur in these models.
\end{abstract}

*Electronic address: htcho@mail.tku.edu.tw

${ }^{\dagger}$ Electronic address: g3180011@tkgis.tku.edu.tw 
Suzuki and Maeda 1] has recently showed that the motions of spinning particles in the Schwarzschild black hole spacetime could be chaotic. This is an important result due to its relevancy to the detection of gravitational waves from black hole coalescences [2, 3], and it has also aroused our interest in considering the chaotic motions of spinning particles.

On the other hand, spinning particle motions have been considered in 4, 5] from a different point of view. The authors there described spinning particles as particles moving in a supersymmetric space, which they called the spinning space, with Grassmann-valued vector variables in addition to the ordinary spacetime coordinates. Hence, the interplay between chaos and spinning particle models involving pseudoclassical mechanics [6] looks rather intriguing.

In this letter we would like to consider the simplest one-dimensional spinning particle model (Eq. (5) below) derived from pseudoclassical mechanical systems. This is just the spinning particle model considered by Berezin and Marinov [7], or the so-called $G_{3}$ model according to the classification scheme in 6]. We hope that the investigation of this simple model will shed some light on the chaotic behaviors of more complicated spinning particle models in higher dimensions or even in curved spaces.

Here we use an analytic method called the Melnikov technique [8, 9, 10] to detect the onset of chaotic motions. Suppose in an integrable system there are homoclinic orbits emanating and terminating at the same unstable fixed point. Time-dependent perturbations are then added to this system. In the perturbed system the stable and unstable orbits split. An integral called the Melnikov function evaluated along the unperturbed homoclinic orbit measures the transversal distance between the perturbed stable and unstable orbits on the Poincaré section. The presence of isolated zeros in the Melnikov function indicates complicated entanglements between the two perturbed orbits and thus the presence of chaotic behaviors.

We start with the bosonic part of our model. We choose the one-dimensional Duffing hamiltonian,

$$
H=\frac{p^{2}}{2}+V(x)
$$

where

$$
V(x)=-x^{2}+x^{4}
$$

The equations for the homoclinic orbit (separatrix) with energy $E=0$ are particularly 
simple,

$$
\begin{aligned}
& x_{s}(t)=\operatorname{sech}(\sqrt{2} t) \\
& p_{s}(t)=-\sqrt{2} \operatorname{sech}(\sqrt{2} t) \tanh (\sqrt{2} t) .
\end{aligned}
$$

This is the main reason we choose the Duffing potential. On the other hand, we expect similar analysis as the one we carry out below can be applied to other potentials with homoclinic or heteroclinic orbits.

To consider pseudoclassical models with Grassmann variables, we concentrate on those with three Grassmann variables $\xi_{1}, \xi_{2}$, and $\xi_{3}$,

$$
H=\frac{p^{2}}{2}+V(x)-\frac{i}{2} \sum_{i, j, k=1}^{3} \epsilon_{i j k} W_{i}(x) \xi_{j} \xi_{k} .
$$

where $W_{i}(x)$ are the potentials for the Grassmann variables. The hamiltonian can be written as 6 ]

$$
H=\frac{p^{2}}{2}+V(x)-\vec{W}(x) \cdot \vec{S}
$$

where the "spin" $\vec{S}$ is defined by

$$
S_{i}=-\frac{i}{2} \epsilon_{i j k} \xi_{j} \xi_{k}
$$

The dynamical equations are

$$
\begin{aligned}
& \frac{d x}{d t}=p, \\
& \frac{d p}{d t}=-V^{\prime}(x)+\vec{W}^{\prime}(x) \cdot \vec{S}, \\
& \frac{d \vec{S}}{d t}=-\vec{W}(x) \times \vec{S},
\end{aligned}
$$

where the prime' denotes $d / d x$. Note that from here on, we work directly with this set of dynamical equations without further reference to the Grassmann nature of $\vec{S}$ as defined in Eq. (17). That is, we do not restrict

$$
S_{i}^{2} \sim 0
$$

Hence, we are dealing with the spinning particle models defined by the dynamical equations rather than the general pseudoclassical systems with Grassmann variables 11]. In effect we just use the pseudoclassical hamiltonians as a convenient and systematic means to derive the dynamical equations for the spinning particles. 


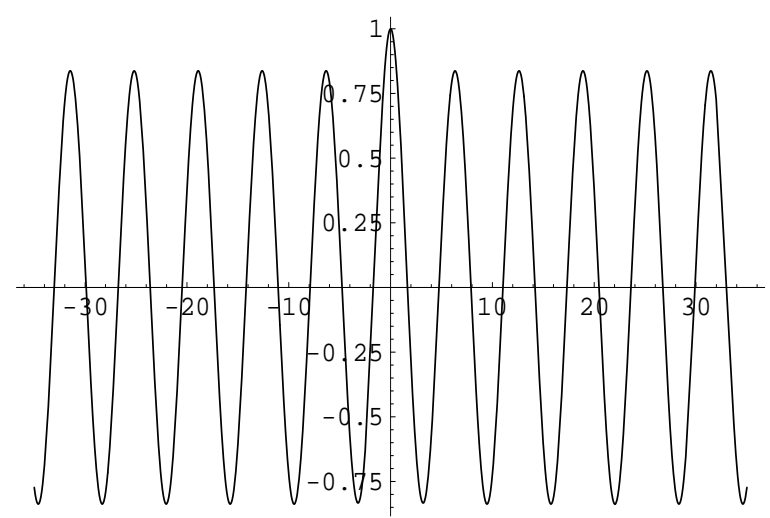

FIG. 1: Time evolution of $\tilde{S}_{1}$ in Eqs. (14)-(16) with the initial conditions as given in Eq. (17).

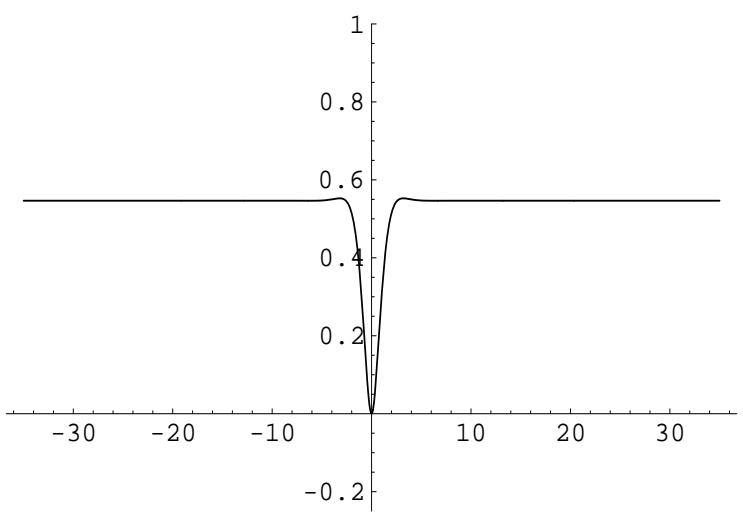

FIG. 2: Time evolution of $\tilde{S}_{2}$ in Eqs. (14)-(16) with the initial conditions as given in Eq. (17).

To detect the onset of chaotic motions in the spinning particle models above, we treat the spin $\vec{S}$ as a perturbation. That is, we assume that

$$
x, p \sim O(1) \text { and } S_{i} \sim O(\epsilon)
$$

for some small parameter $\epsilon$. Then the additional spin terms in Eq. (9) have the form of time-dependent perturbations which may trigger chaotic behaviors.

To make the discussion more concrete we first take a simple form for the potentials $\vec{W}(x)$,

$$
W_{1}=x ; W_{2}=1 ; W_{3}=0 .
$$

We shall return to the consideration of more general $\vec{W}(x)$ below. To obtain the lowest order solutions of $S_{i}$, we just replace $\vec{W}(x)$ in the spin dynamical equations (Eq. (10) ) by $\vec{W}\left(x_{s}(t)\right)$, that is,

$$
\frac{d \tilde{S}_{1}}{d t}=-\tilde{S}_{3}
$$




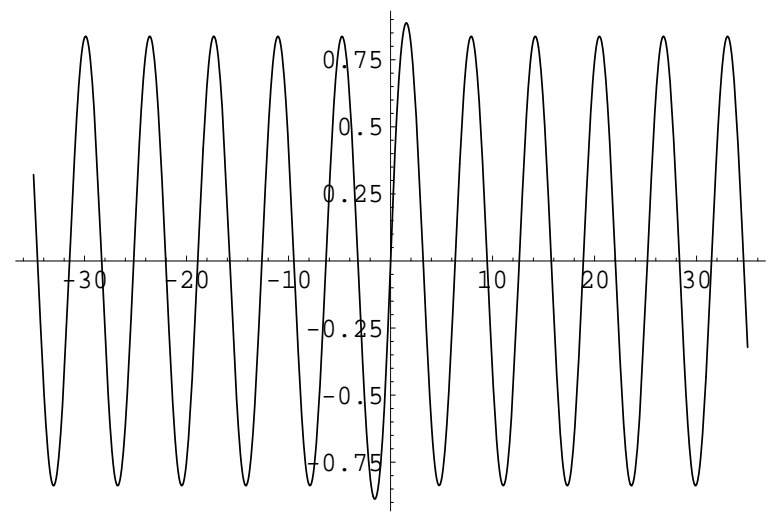

FIG. 3: Time evolution of $\tilde{S}_{3}$ in Eqs. (14)-(16) with the initial conditions as given in Eq. (17).

$$
\begin{aligned}
\frac{d \tilde{S}_{2}}{d t} & =\operatorname{sech}(\sqrt{2} t) \tilde{S}_{3} \\
\frac{d \tilde{S}_{3}}{d t} & =\tilde{S}_{1}-\operatorname{sech}(\sqrt{2} t) \tilde{S}_{2}
\end{aligned}
$$

where we have written $S_{i}=\epsilon \tilde{S}_{i}$. We can numerically solve this set of differential equations quite easily using, for example, Mathematica. The solutions with the initial conditions

$$
\tilde{S}_{1}(0)=1 \quad ; \quad \tilde{S}_{2}(0)=\tilde{S}_{3}(0)=0
$$

are plotted in Figs. 1] 3, $\tilde{S}_{1}$ and $\tilde{S}_{3}$ are oscillatory, while $\tilde{S}_{2}$ is mostly constant except for a small region near $t=0$. Note that the choice of the initial conditions here is quite arbitrary. We have checked the behaviors of the spins for other sets of initial conditions like $\tilde{S}_{1}(0)=0$; $\tilde{S}_{2}(0)=1 ; \tilde{S}_{3}(0)=0$ and $\tilde{S}_{1}(0)=0 ; \tilde{S}_{2}(0)=0 ; \tilde{S}_{3}(0)=1$, and similar results like those in Figs. 1],3] are obtained.

It is well known that external oscillatory perturbations may induce chaotic behaviors. We can see that this is indeed the case here by calculating the Melnikov function [8, 9],

$$
\begin{aligned}
M\left(t_{0}\right) & =\int_{-\infty}^{\infty} d t p_{s}\left(t+t_{0}\right) \vec{W}^{\prime}\left(x_{s}(t)\right) \cdot \vec{S}(t) \\
& =-\int_{-\infty}^{\infty} d t \sqrt{2} \operatorname{sech}\left[\sqrt{2}\left(t+t_{0}\right)\right] \tanh \left[\sqrt{2}\left(t+t_{0}\right)\right] S_{1}(t),
\end{aligned}
$$

where $t_{0}$ is used to parametrize the location along the unperturbed homoclinic orbit. $M\left(t_{0}\right)$ is plotted in Fig. 4. The Melnikov function measures the transveral distance on the Poincaré section between the perturbed stable and unstable orbits passing through the unstable fixed point. The infinite number of zeros as seen in Fig. 4 indicates the entanglement of the two orbits, and this signals the occurrence of chaotic behavior for the perturbed homoclinic 


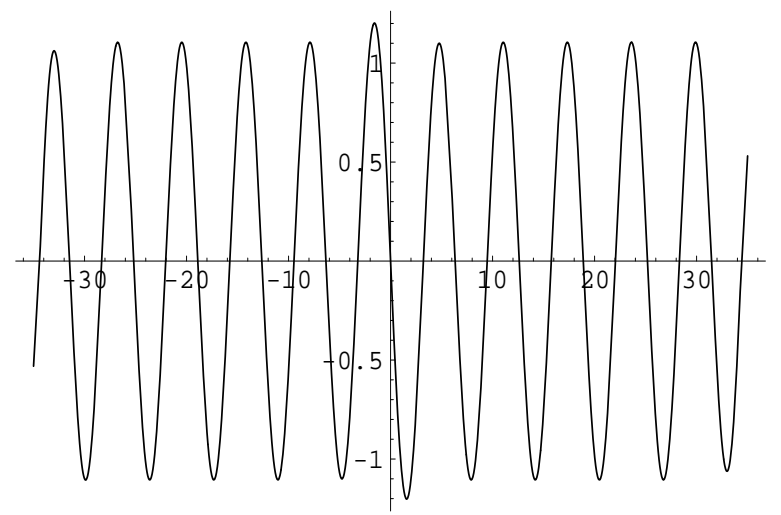

FIG. 4: Melnikov function of the spinning particle model in Eq. (6) with the potentials for the spins $\vec{W}(x)$ as given in Eq. (13) .

orbits. When the perturbation terms, or the spin terms here, become larger and larger, the chaotic behavior will spread to other parts of the phase space.

For more general $\vec{W}(x)$ potentials, we can argue that similar chaotic behaviors as those with the simple potential in Eq. (13) discussed above will again occur. In this case the Melnikov function is still given by Eq. (18) but with general $\vec{W}(x)$. The key point to the argument is to consider the large $t_{0}$ behavior of $M\left(t_{0}\right)$. This is just the region near the unstable fixed point where complicated homoclinic entanglements may occur. Now in the integrand in Eq. (18),

$$
p_{s}\left(t+t_{0}\right)=-\sqrt{2} \operatorname{sech}\left[\sqrt{2}\left(t+t_{0}\right)\right] \tanh \left[\sqrt{2}\left(t+t_{0}\right)\right]
$$

which is exponentially small except for

$$
t \sim-t_{0}
$$

Hence, to estimate $M\left(t_{0}\right)$ for large $t_{0}$, we need only to consider the behaviors of $\vec{W}^{\prime}\left(x_{s}(t)\right)$ and $\vec{S}(t)$ for $t \sim-t_{0}$ or for large $|t|$.

For large $|t|, x_{s}(t)$ is also exponentially small. Suppose, for small $x$,

$$
W_{i} \simeq a_{i}+b_{i} x+\cdots,
$$

where $a_{i}$ and $b_{i}$ are constants. Then, for large $|t|, W_{i}$ can be approximated by $a_{i}$, and the 
spin equations become

$$
\frac{d}{d t}\left(\begin{array}{c}
\tilde{S}_{1} \\
\tilde{S}_{2} \\
\tilde{S}_{3}
\end{array}\right) \simeq\left(\begin{array}{ccc}
0 & a_{3} & -a_{2} \\
-a_{3} & 0 & a_{1} \\
a_{2} & -a_{1} & 0
\end{array}\right)\left(\begin{array}{c}
\tilde{S}_{1} \\
\tilde{S}_{2} \\
\tilde{S}_{3}
\end{array}\right)
$$

The eigenvalues of the matrix above are 0 and $\pm i a$, where $a=\sqrt{a_{1}^{2}+a_{2}^{2}+a_{3}^{2}}$. The solutions of $\tilde{S}_{i}$ are therefore linear combinations of $\sin (a t), \cos (a t)$, and some constants. Except for the case when all $a_{i}=0$, some of the components $\tilde{S}_{i}$ must be oscillatory.

Similarly, for large $|t|$,

$$
W_{i}^{\prime} \simeq b_{i}
$$

Hence the Melnikov function for large $t_{0}$ can be written approximately as a linear combination of

$$
\begin{aligned}
& M_{1}\left(t_{0}\right)=\int_{-\infty}^{\infty} d t p_{s}\left(t+t_{0}\right)=0, \\
& M_{2}\left(t_{0}\right)=\int_{-\infty}^{\infty} d t p_{s}\left(t+t_{0}\right) \sin (a t)=-\frac{\pi a}{\sqrt{2}} \operatorname{sech}\left(\frac{\pi a}{2 \sqrt{2}}\right) \cos \left(a t_{0}\right), \\
& M_{3}\left(t_{0}\right)=\int_{-\infty}^{\infty} d t p_{s}\left(t+t_{0}\right) \cos (a t)=-\frac{\pi a}{\sqrt{2}} \operatorname{sech}\left(\frac{\pi a}{2 \sqrt{2}}\right) \sin \left(a t_{0}\right),
\end{aligned}
$$

where we have used the fact that $p_{s}(t)$ is odd in $t$. The Melnikov function $M\left(t_{0}\right)$ for general $\vec{W}(x)$ potentials with the small $x$ behavior as shown in Eq. (21) is therefore an oscillatory function with infinity number of simple zeros at large $t_{0}$. This is sufficient to indicate the occurrence of chaotic behaviors for these general spinning particle models.

In summary, we have indicated, using the method of Melnikov functions, the presence of homoclinic entanglements and thus the onset of chaos in one-dimensional spinning particle models with a Duffing potential and some general potentials for the spins. We expect similar results for other potentials with homoclinic or heteroclinic orbits. Moreover, the models we consider in this letter are derived from one-dimensional pseudoclassical systems with three Grassmann variables, so we also expect chaos to occur for spinning particle models derived from systems with three or more Grassmann variables where more degrees of freedom are involved.

As we have mentioned at the beginning, chaotic behaviors do occur for the motions of spinning particles in a Schwarzschild black hole spacetime which can be described by a 
supersymmetric theory. This shows that we should extend our investigation of chaos in spinning particle models to higher dimensions as well as to models in curved spacetimes. We plan to do that in our future publications.

We would like to thank C.-C. Chen for useful discussions. This work was supported by the National Science Council of the Republic of China under contract number NSC89-2112M-032-021.

[1] S. Suzuki and K. Maeda, Phys. Rev. D. 55, 4848 (1997).

[2] J. Levin, Phys. Rev. Lett. 84, 3515 (2000).

[3] N. J. Cornish, Phys. Rev. D. 64, 084011 (2001).

[4] R. H. Rietdijk and J. W. van Holten, Class. Quantum Grav. 7, 247 (1990).

[5] R. H. Rietdijk and J. W. van Holten, Class. Quantum Grav. 10, 575 (1993).

[6] R. Casalbuoni, Nuovo Cim. 33A, 389 (1976).

[7] T. A. Berezin and M. S. Marinov, Ann. Phys. (N. Y.) 104, 336 (1977).

[8] V. K. Melnikov, Trans. Moscow Math. Soc. 12, 1 (1963).

[9] P. Holmes, Phys. Rept. 193, 137 (1990).

[10] L. Bombelli and E. Calzetta, Class. Quantum Grav. 9, 2573 (1992).

[11] R. Heumann and N. S. Manton, Ann. Phys. (N.Y.) 284, 52 (2000). 